\title{
A mathematical model of actin filament turnover for fitting FRAP data
}

\author{
Aliaksandr A. Halavatyi • Petr V. Nazarov • \\ Ziad Al Tanoury • Vladimir V. Apanasovich • \\ Mikalai Yatskou $\cdot$ Evelyne Friederich
}

Received: 9 June 2009/Revised: 24 August 2009/Accepted: 19 October 2009/Published online: 18 November 2009

(C) European Biophysical Societies' Association 2009

\begin{abstract}
A novel mathematical model of the actin dynamics in living cells under steady-state conditions has been developed for fluorescence recovery after photobleaching (FRAP) experiments. As opposed to other FRAP fitting models, which use the average lifetime of actins in filaments and the actin turnover rate as fitting parameters, our model operates with unbiased actin association/dissociation rate constants and accounts for the filament length. The mathematical formalism is based on a system of stochastic differential equations. The derived equations were validated on synthetic theoretical data generated by a stochastic simulation algorithm adapted for the simulation of FRAP experiments. Consistent with experimental findings, the results of this work showed that (1) fluorescence recovery is a function of the average filament length, (2) the F-actin turnover and the FRAP are accelerated in the presence of actin nucleating proteins, (3) the FRAP curves may exhibit both a linear and non-linear behaviour depending on the parameters of actin polymerisation, and
\end{abstract}

The more you see: spectroscopy in molecular biophysics.

A. A. Halavatyi · Z. Al Tanoury - M. Yatskou ·

E. Friederich $(\square)$

Cytoskeleton and Cell Plasticity Laboratory,

Life Sciences Research Unit, Faculty of Science,

Technology and Communication, University of Luxembourg,

162 A ave de la Faïencerie, Campus Limpertsberg,

Luxembourg 1511, Luxembourg

e-mail: evelyne.friederich@uni.lu

\section{P. V. Nazarov}

Microarray Center, Centre de Recherche Public Santé,

84, Rue Val Fleuri, Luxembourg 1526, Luxembourg

V. V. Apanasovich

Department of Systems Analysis, Belarusian State University,

4 Nezavisimosti Avenue, Minsk 220030, Belarus
(4) our model resulted in more accurate parameter estimations of actin dynamics as compared with other FRAP fitting models. Additionally, we provide a computational tool that integrates the model and that can be used for interpretation of FRAP data on actin cytoskeleton.

Keywords FRAP · Mathematical model ·

Polymerisation · Actin · Filament

\section{Introduction}

Actin polymerisation is an important mechanism in many intracellular processes, including cell morphogenesis and motility, in physiological situations and disease (see Pollard and Borisy 2003 for review). Actin is an asymmetric protein able to self-assemble into proteanous filaments with structurally and biochemically distinct ends. The rates of actin association and dissociation at the so-called barbed end of the actin filament are ten times higher than those at the pointed end. Actin polymerisation is regulated by actin accessory proteins, which cause actin nucleation and capping, severing or branching of actin filaments (Giganti and Friederich 2003; Giganti et al. 2005; Mogilner 2009). Because structure and dynamics of the actin cytoskeleton differ in distinct cellular sub-domains, in vitro assays can not fully reproduce actin turnover in living cells, but nevertheless provide useful insight into actin polymerisation reactions. Actin polymerisation kinetics in living cells can be investigated using fluorescent actin analogues and laserassisted confocal microscopy, which allows visualisation of labelled proteins and provides information on their spatial distribution.

Various experimental protocols have been set-up for the quantification of the dynamic and structural properties of 
molecular complexes, characterising distances between molecules, rates of biochemical reactions and mobility parameters. Laser-assisted, confocal microscopy approaches, such as fluorescence recovery after photobleaching (FRAP) (Carrero et al. 2003), fluorescence loss in photobleaching (FLIP) (Phair and Misteli 2000) and fluorescence localisation after photobleaching (FLAP) (Dunn 2002), make use of fluorescent probes which are bleached under a high intensity laser pulse. The fluorescence non-homogeneity generated by bleaching decreases due to the diffusion of non-fluorescent molecules out of the bleached area and of fluorescent molecules into the bleached area. The rate of diffusion can be influenced by molecular interactions in and out of the bleached region. Measuring exchange rates of bleached molecules by fluorescent ones provides quantitative information about molecular reactions and mobility of proteins in living cells.

In FRAP experiments, relatively small regions of the cell are bleached at once, while molecules in other cellular areas remain fluorescently active. Due to diffusion and transport processes, unbleached proteins enter into the bleached spot, participating in biochemical reactions along with bleached molecules. In most cases the time evolution of the average fluorescence intensity in the bleached spot, which is proportional to the number of fluorescence molecules for a relatively low concentration of labelled particles, is analysed. The rate and shape of the fluorescence intensity recovery in time depend on the underlying processes and their numerical characteristics, such as diffusion coefficients and reaction rate constants. A mathematical model may then be applied to extract relevant parameter values from experimental FRAP data. The equations or simulation algorithms of such models account for the recovery limiting factors and may differ in the level of details and properties of studied biomolecules.

The existing FRAP fitting models can be used for the analysis of protein diffusion (Ellenberg et al. 1997; Soumpasis 1983) and binding reactions for one and several binding states, including cases where diffusion is a limiting factor for binding processes (Carrero et al. 2004; Mueller et al. 2008; Sprague et al. 2004). This methodology provides important quantitative information on molecular dynamics in vivo, such as diffusion constants, rate constants of protein (un)binding, (im)mobile fraction(s) of proteins, spatial localisation of the fluorescent protein analogues. Only a few studies at present used FRAP, associated with mathematical modelling, to gain quantitative information about actin polymerisation in living cells. Some of these studies have used binding approximation (Carrero et al. 2004; McDonald et al. 2006) to measure actin turnover in the nucleus or in cytoplasm (Campbell and Knight 2007). The model of Tardy et al. (1995) was established specifically for FRAP experiments with actin filaments and was applied for the fitting of corresponding datasets. The model accounts for several important factors, such as diffusion of actin monomers, but operates with a rate of exchange between two pools of actins to describe polymerisation reactions. In the work of Lai et al. (2008), probabilities of conversion between filamentous and monomeric forms of actin were assumed to be functions of the distance from the leading edge. This assumption allowed obtaining an accurate fit for measured recoveries, but the mentioned dependences were not quantitatively linked to the spatial properties of filaments. Indeed, in all these models, the polarity and length of actin filaments as well as the dynamics of their pointed and barbed ends are neglected. Thus, they do not allow using values of fitted parameters as measures of actin gain/loss on the filament ends.

Direct visualisation of filaments and total internal reflection fluorescence microscopy (Fujiwara et al. 2002) together with stochastic simulation (Matzavinos and Othmer 2007; Roland et al. 2008) have shown that both experimental procedures and models for data analysis should account for the length of filaments to estimate polymerisation rates. In addition, the filament length is an important biophysical parameter of actin cytoskeleton mechanics. Results of FRAP experiments are potentially able to reflect the structure and turnover dynamics of actin filaments, but no dedicated mathematical treatment of FRAP data obtained with fluorescent actin analogues is available at the present date in the context of dynamic and structural properties of actin filaments. Thus, the formulation of new mathematical issues, which can be used to estimate the kinetic rate constants and filament length, are needed for FRAP studies of actin polymerisation.

In this paper, we present a novel mathematical model that can be used to fit the FRAP data obtained with GFPactin and to calculate the FRAP response on actin polymerisation system with the parameters accounting for the average filament length $(\langle L\rangle)$ and association/dissociation at the barbed and pointed ends of actin filaments $\left(k_{b}^{+}, k_{b}^{-}, k_{p}^{+}, k_{p}^{-}\right)$. Unlike the previously published models, we show that FRAP curves may exhibit both non-linear and linear behaviours under some specific parametric configurations governed by the coefficients of elongation $(v)$ and diffusivity $(D)$, the parameters that are defined by the kinetic rate constants.

\section{Methodology}

Kinetic model for fitting equation

Actin polymerisation leads to formation of long linear double helix filaments, $1-10 \mu \mathrm{m}$, with actin subunits spiralbound around the axis of the filament (Pollard and Borisy 2003). Both strands of the helix have the same polarity, so 
the two ends of the filament are structurally different, termed barbed (BE) and pointed ends (PE). Often a mathematical model of a filament is approximated by a linear chain of actin subunits (Fass et al. 2008). Under steady-state conditions, the average length $\langle L\rangle$ of filaments does not change in time and the actin dynamics of individual actin filaments is governed by treadmilling process, defining the actin turnover time (Matzavinos and Othmer 2007). We would like to deduce a mathematical kinetic model to describe such an assay to be applied to FRAP experiments.

The scheme of the system formalization is shown in Fig. 1a. A pool of free globular (G-) and filamentous (F-) actins with the constant concentrations $c_{m}$ and $c_{f}$ in time and volume is considered. An exchange between F-actin and G-actin is controlled by the turnover process, which is

$\mathbf{a}$
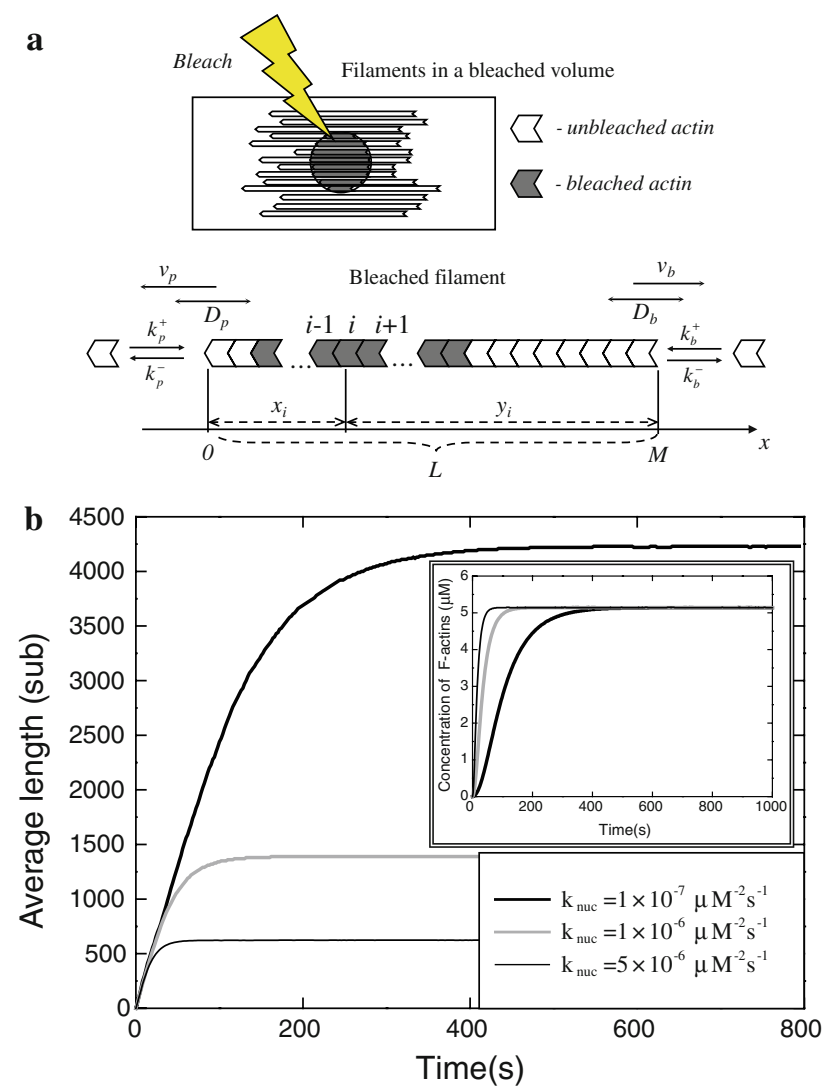

Fig. 1 Schematic diagrams and model calculations for actin polymerisation dynamics. a Schematic representation of a FRAP experiment on GFP-actin. Actin filaments are partially or completely bleached depending on the size of the bleached spot. Parameters are the association and dissociation rate constants at the $\mathrm{BE}$ and $\mathrm{PE}$ $k_{b}^{+}, k_{b}^{-}, k_{p}^{+}, k_{p}^{-}$, the average length of filaments $\langle L\rangle$, and the concentration of F-actin $c_{f}$. b Results of stochastic simulation of artificial actin polymerisation systems at different nucleation rates. The inset represents the increase in $\mathrm{F}$-actin concentration as a function of time. Parameters are $k_{b}^{+}=10.0 \mu \mathrm{M}^{-1} \mathrm{~s}^{-1}, k_{b}^{-}=1.0 \mathrm{~s}^{-1}, k_{p}^{+}=$ $0.5 \mu \mathrm{M}^{-1} \mathrm{~s}^{-1}, k_{p}^{-}=8.0 \mathrm{~s}^{-1}$, and the initial concentration of actin monomers is $6 \mu \mathrm{M}$ determined by the rates of actin association/dissociation at the BE and PE: $\left(k_{b}^{+}, k_{b}^{-}, k_{p}^{+}, k_{p}^{-}\right)$. We assume the following:

1. Since G-actins diffuse rapidly (McGrath et al. 1998), their spatial distribution is homogeneous.

2. Actin filaments do not diffuse. This is appropriate for many cell compartments because filaments are crosslinked into networks and linked by protein complexes to the plasma membrane.

3. Contribution of actin spontaneous nucleation in steady state is insignificant (Roland et al. 2008).

4. A spatial concentration of actin filaments is homogeneous.

5. The bleached area could be approximated by a cylinder with an axis perpendicular to the sample surface. Fluorescence intensity is measured in the thin layer inside this cylinder perpendicular to its axis by confocal microscope.

6. Disassembled actins leave a bleach spot instantly and irreversibly. This assumption is typically acceptable for modelling of the FRAP recovery in the reaction dominant regime, when a bleached spot has a rather small size and diffusion is fast compared to association/dissociation kinetics and the time scale of the FRAP experiment (Sprague et al. 2004).

At steady state, the full fluorescence recovery of the modelled system is merely due to the gain and loss of actin subunits. After laser illumination of a cell area, actin filaments may be composed of bleached and unbleached actin subunits whose distribution along the average filament length $\langle L\rangle$ is defined by the size of the bleached region. The resulting FRAP curve is calculated as a superposition of the probability events that any bleached actin disassembles before the time $t$ at either end.

We applied a theory of stochastic processes to simulate the turnover of bleached/unbleached actins in the bleached area. The balance equation for the probability $p_{i}(t)$ of the chemical system state $i$ in a closed volume can be given by the following master equation (van Kampen 2007)

$\frac{\mathrm{d} p_{i}(t)}{\mathrm{d} t}=\sum_{j=1}^{J}\left(-W_{i j} p_{i}(t)+W_{j i} p_{j}(t)\right)$,

where $J$ is the total number of possible states; $W_{i j}, W_{j i}$ are the transition probability per time unit from the states $i$ to $j$ and vice versa.

Without loss of generality, we start from consideration of a single bleached subunit in filament. Then, in Eq. 1 the state $i$ is the position of the bleached subunit in relative to the PE. In the absence of the reactions at the $\mathrm{BE}$, the state $i$ can exchange with the neighbouring states $(i-1)$ and $(i+1)$ via association and dissociation reactions at the PE (Fig. 1a). 
The corresponding transition probabilities are $W_{i(i+1)}=$ $W_{(i-1) i}=k_{p}^{+} c_{m}$ and $W_{i(i-1)}=W_{(i+1) i}=k_{p}^{-}$(Gadgil et al. 2005), and Eq. 1 becomes

$$
\begin{gathered}
\frac{\mathrm{d} p_{i}(t)}{\mathrm{d} t}=-\left(k_{p}^{+} c_{m}+k_{p}^{-}\right) p_{i}(t)+k_{p}^{+} c_{m} p_{i-1}(t)+k_{p}^{-} p_{i+1}(t) \\
i=1,2, \ldots, M
\end{gathered}
$$

where $M$ is the maximum number of actin subunits in filament and $p_{0}=0$. Equation 2 represents a set of $M$ coupled differential equations. Assuming rather long filaments of $1 \mu \mathrm{m}$ or more $(M>400)$, we can replace the probabilities $p_{i}(t)$ with a probability density function $\rho(x, t)$ of bleached actin subunit positions along filament. Applying a number of proper transformations, $p_{i}(t)=\rho\left(x_{i}, t\right) \delta, \rho\left(x_{i}, t\right)+\rho\left(x_{i+1}, t\right) \approx 2 \rho\left(x_{i}, t\right), \rho\left(x_{i+1}, t\right)$ $-\rho\left(x_{i}, t\right) \approx \frac{\partial \rho\left(x_{i}+0.5 \delta, t\right)}{\partial x} \delta$, where $\delta$ is a distance between two neighbouring subunits in the filament and $x_{i}=i \times \delta$ is a position of the $i$-th bleached subunit in the filament, the system of Eq. 2 is approximated by a single continuous differential equation at the limit $M \rightarrow \infty$ as

$\frac{\partial \rho(x, t)}{\partial t} \approx \frac{k_{p}^{-}+k_{p}^{+} c_{m}}{2} \delta^{2} \frac{\partial^{2} \rho(x, t)}{\partial x^{2}}-\left(k_{p}^{+} c_{m}-k_{p}^{-}\right) \delta \frac{\partial \rho(x, t)}{\partial x}$,

where $\rho(0, t)=0$ is an absorbing boundary condition (van Kampen 2007). By introducing the parameter-dependent coefficients $v_{p}=\left(k_{p}^{+} c_{m}-k_{p}^{-}\right) \delta$ and $D_{p}=\frac{k_{p}^{-}+k_{p}^{+} c_{m}}{2} \delta^{2}$ we obtain

$\frac{\partial \rho(x, t)}{\partial t}=D_{p} \frac{\partial^{2} \rho(x, t)}{\partial x^{2}}-v_{p} \frac{\partial \rho(x, t)}{\partial x}$.

Equation 4 is a Fokker-Plank equation used elsewhere to describe a diffusion type process (Gardiner 2004). The term $v_{p} \frac{\partial \rho(x, t)}{\partial x}$ of Eq. 4 evaluates the average growth of the PE, and its coefficient $v_{p}$ is the elongation rate of the PE. The value of $v_{p}$ is positive when assembly reactions dominate over disassembly reactions leading to an elongation of the PE. It is negative in the opposite case. The term $D_{p} \frac{\partial^{2} \rho(x, t)}{\partial x^{2}}$ describes a stochastic fluctuation of the filament length in time; its coefficient $D_{p}$ has a meaning of the diffusion coefficient when Eq. 4 is treated as a one-dimensional diffusion. The variance $\sigma_{p}^{2}$ of the filament PE length is time-dependent

$\sigma_{p}^{2}=2 D_{p} t$

The solution of Eq. 4 satisfying the boundary condition (assumption 6) for a bleached actin subunit situated in the position $x_{0}$ at time $t=0$ is given by Eq. 6:

$\rho_{x_{0}}(x, t)=\frac{1}{2 \sqrt{\pi D_{p} t}}\left(\mathrm{e}^{-\frac{\left(x-x_{0}-v_{p} t\right)^{2}}{4 D_{p} t}}-\mathrm{e}^{-\frac{\left(x+x_{0}-v_{p} t\right)^{2}}{4 D_{p} t}-\frac{v p x_{0}}{D_{p}}}\right)$.

The first term in Eq. 6 represents the equation of diffusion with a drift in the unlimited area. The second term accounts for the irreversible loss of the bleached particles when they dissociate at the filament ends.

Then, we calculate the probability function $P_{p}\left(x_{0}, t\right)$ of the random event that a bleached actin subunit at a fixed initial position $x_{0}$ is still associated with filament (or would not disassemble from the PE) by the time $t$ after the bleach. This leads to integration of the probability density function $\rho_{x_{0}}(x, t)$ over the infinite range of the variable $x$ :

$$
\begin{aligned}
P_{p}\left(x_{0}, t\right) & =\int_{0}^{\infty} \rho_{x_{0}}(x, t) \mathrm{d} x \\
& =\Phi\left(\frac{x_{0}+v_{p} t}{\sqrt{2 D_{p} t}}\right)-\mathrm{e}^{-\frac{v_{p} x_{0}}{D_{p}}} \Phi\left(\frac{v_{p} t-x_{0}}{\sqrt{2 D_{p} t}}\right),
\end{aligned}
$$

where $\Phi(u)=\frac{1}{\sqrt{2 \pi}} \int_{-\infty}^{u} \mathrm{e}^{-t^{2} / 2} \mathrm{~d} t$ is the cumulative distribution function for the standard normal distribution. If $D_{p}$ is close to 0 , the probability defined by Eq. 7 converges to unity.

Using a similar approach, we can calculate the probability function $P_{b}\left(y_{0}, t\right)$ for the $\mathrm{BE}$ in the absence of the reactions at the $\mathrm{PE}$

$P_{b}\left(y_{0}, t\right)=\Phi\left(\frac{y_{0}+v_{b} t}{\sqrt{2 D_{b} t}}\right)-\mathrm{e}^{-\frac{v_{b} y_{0}}{D_{b}}} \Phi\left(\frac{v_{b} t-y_{0}}{\sqrt{2 D_{b} t}}\right)$,

where $v_{b}=\left(k_{b}^{+} c_{m}-k_{b}^{-}\right) \delta$ is the elongation rate at the $\mathrm{BE}$, $D_{b}=\frac{k_{b}^{+} c_{m}+k_{b}^{-}}{2} \delta^{2}$ is the diffusivity coefficient at the BE and $y_{0}$ is the position of a bleached actin subunit relative to the BE.

Assuming independent reactions at the $\mathrm{BE}$ and $\mathrm{PE}$ and replacing $y_{0}$ by $\left(\langle L\rangle-x_{0}\right)$, the total probability function for the bleached actin subunit to reside in the filament at time $t$ is a product of the probability functions

$P\left(x_{0}, t\right)=P_{p}\left(x_{0}, t\right) P_{b}\left(\langle L\rangle-x_{0}, t\right)$.

Integrating over all initial states, $x_{0}$, of the bleached actin subunits in filament, we obtain

$P(t)=\int_{0}^{\langle L\rangle} P\left(x_{0}, t\right) \varphi\left(x_{0}\right) \mathrm{d} x_{0}$,

where $\varphi\left(x_{0}\right)$ is a normalised distribution of the bleached states $x_{0}$ for all parts of the filaments inside the bleached region at the bleach moment $(t=0)$ and $\int_{0}^{\langle L\rangle} \varphi(\xi) \mathrm{d} \xi=1$. When the ends of filaments are not bunched in the specific cell region, their various parts are situated randomly inside the bleached spot and $\varphi\left(x_{0}\right)=\langle L\rangle^{-1}$.

Finally, accounting for the steady-state concentrations of free actin proteins $c_{m}$ and F-actins $c_{f}$ we obtain the normalised FRAP recovery of the G- and F-actin 


$$
\begin{aligned}
\operatorname{FRAP}(t) & =\frac{c_{m}}{c_{m}+c_{f}}+\frac{c_{f}}{c_{m}+c_{f}}(1-P(t)) \\
& =1-\frac{c_{f}}{c_{m}+c_{f}} P(t)
\end{aligned}
$$

The FRAP model was programmed in Mathematica 6.0 (Wolfram Research), and the corresponding routine actinfrap.nb is available at the website http://actinsim. uni.lu/.

Stochastic simulation algorithm

To test the derived kinetic model, we applied a stochastic simulation algorithm (SSA), similar to that reported in Fass et al. (2008) and Roland et al. (2008). This stochastic approach provides precise information on the dynamics of G- and F-actin, including the structural compositions of actin filaments (Halavatyi et al. 2009). We adapted the SSA for the simulation of a FRAP experiment, considering bleached and unbleached actin subunits as independent molecular species. Synthetic datasets were generated to test and validate the deduced fitting equation. In particular, we estimated the time-resolved evolution of the F-actin concentration and the growth of the average filament length for the non-equilibrium initial system states and the standard sets of reaction parameters. All FRAP curves were simulated for the steady-state phase and covered a broad range of the model parameters $\left(\langle L\rangle, c_{f}, k_{b}^{+}, k_{b}^{-}, k_{p}^{+}, k_{p}^{-}\right)$.

\section{Results and discussion}

Laser-assisted confocal microscopy is frequently used to visualise and analyse the dynamics of the actin cytoskeleton in living cells. Actin filaments of specific sub-regions of cells are grouped and cross-linked into bundles or networks. Such a bulk of filaments does not allow tracking individual filaments and changes in their structures. However, both filament lengths and their turnover properties are important characteristics of overall actin dynamics in cells. As shown above, FRAP curves depend on these quantities, which allow measuring cytoskeleton behaviour in different regions of the cell without changing cell properties.

Filaments may be oriented either in parallel to each other in bundles, for example at focal adhesion sites, or organised into branched networks in a lamellipodium. For a single filament only a part that is situated inside the bleached cylinder at the bleach moment may be bleached then. Because the resulting recovery is influenced by the spatial organisation of all the filaments in the region rather than the positions of individual filaments, we could account for the filament orientation relative to the bleached spot by the distribution function $\varphi\left(x_{0}\right)$ of bleached actin protomers along the filament (see Eq. 10). For most cases of bulk cytoskeleton in the cell regions where the ends of the filaments are not gathered, numbers of protomers corresponding to the different $x_{0}$ values inside the bleached spot are approximately equal, and this function can be represented by a uniform distribution.

Before the bleach, the system is in equilibrium, i.e. the probabilities for assembly and disassembly reactions at both ends of the filament are equal $\left(k_{b}^{+} c_{m}+k_{p}^{+} c_{m}=k_{b}^{-}+k_{p}^{-}\right)$, the critical monomer concentration of actin or the steadystate monomer concentration showing the total equilibrium between association and dissociation processes is calculated as

$c_{m}=\frac{k_{b}^{-}+k_{p}^{-}}{k_{b}^{+}+k_{p}^{+}}$

Then the elongation rates $v_{b}, v_{p}$ and diffusivity coefficients $D_{b}, D_{p}$ defined by the rate constants are

$v_{b}=-v_{p}=\frac{k_{b}^{+} k_{p}^{-}-k_{p}^{+} k_{b}^{-}}{k_{b}^{+}+k_{p}^{+}} \delta$,

$D_{b}=\frac{2 k_{b}^{+} k_{b}^{-}+k_{b}^{+} k_{p}^{-}+k_{p}^{+} k_{b}^{-}}{2\left(k_{b}^{+}+k_{p}^{+}\right)} \delta^{2}$,

$D_{p}=\frac{2 k_{p}^{+} k_{p}^{-}+k_{b}^{+} k_{p}^{-}+k_{p}^{+} k_{b}^{-}}{2\left(k_{b}^{+}+k_{p}^{+}\right)} \delta^{2}$.

Assuming independent reactions at the $\mathrm{BE}$ and $\mathrm{PE}$ and Eq. 5, we can estimate the variance $\sigma^{2}$ of the filament length as

$\sigma^{2}=\sigma_{b}^{2}+\sigma_{p}^{2}=2\left(D_{b}+D_{p}\right) t=2\left(k_{b}^{-}+k_{p}^{-}\right) \delta^{2} t$

Coefficients $v_{p}$ and $v_{b}$ are of opposite sign and equal absolute value, henceforth denoted as $v$, indicating that shortening from the PE will necessarily be compensated by growth of the BE. In the presence of any capped end, e.g. by a $\mathrm{BE}$ capper, the elongation rate $v$ and the diffusivity coefficient $D_{b}$ are 0 . However, $D_{p} \neq 0$, and therefore the fluorescence recovery is not abolished. In the absence of capped ends, the elongation coefficient $v$ is 0 only if (G-)actin critical concentrations at both filament ends, which are the local equilibrium conditions for $\mathrm{BE}$ and $\mathrm{PE}$, are equal $\left(c_{m, b} \approx c_{m, p}\right)$; then the following relation for the rate constants is relevant:

$\left|\frac{k_{b}^{-}}{k_{b}^{+}}-\frac{k_{p}^{-}}{k_{p}^{+}}\right|=\left|c_{m, b}-c_{m, p}\right| \approx 0$.

The fluorescence recovery relies on stochastic fluctuations of the filament length, characterised by the coefficients $D_{b}$ and $D_{p}$, and exhibits an exponential-like curve.

If the value of $v$ increases, the recovery is in the regime of intensive dissociation via the PE, and the FRAP exhibits 
a linear-like curve. Shortening of the PE is observed during the treadmilling process, and the number of disassembled actins may be estimated as the product of $v \times t$. This effect dominates over disassembly due to diffusivity, characterised by standard deviation $\sigma$, increasing as a square root of the time $(\sigma \sim \sqrt{t}$; see Eq. 14). The slope of the normalised recovery in the linear regime is proportional to the ratio of the elongation rate $v$ and the mean filament length $\langle L\rangle$. For such a treadmilling regime, bleached actins will dissociate mostly at the PE of filaments. The corresponding equation for linear FRAP recovery can be easily derived if the $D_{p}$ value in Eq. 4 is set to 0 and the equations are solved with the same boundary conditions.

The following actin polymerisation reactions were implemented in the SSA to generate the synthetic data: (1) spontaneous and/or stimulated nucleation of actin filaments, (2) actin association at filament ends, (3) actin dissociation at filament ends. The reaction rates may vary depending on the actin-accessory proteins in the cells and cell environment (Lan and Papoian 2008). For example, the actin nucleation mechanism may be accelerated by formin, capping protein, or by filament branching from a 'mother' filament by the Arp2/3 complex (Pollard and Borisy 2003). The protein cofilin enhances filament depolymerisation by increasing the dissociation rate of ADP-actin from the PE (Van Troys et al. 2008). The actin turnover is governed by actin subunit gain and loss at the BE and PE, respectively. We took the values of the corresponding reaction rate constants close to those in vitro to generate the synthetic data (Kuhn and Pollard 2005). The rate of actin dissociation at the PE was increased intentionally to simulate the effect of cofilin.

Taking a physiological range for parameters describing polymerisation mechanisms, we simulated the time evolution of the filament network from the pool of actin monomers. Our simulations were repeated for several nucleation rate constants to estimate further the effect of nucleating proteins on fluorescence recovery. We modelled three systems characterised by the nucleation rate constants $k_{\text {nuc }}=1 \times 10^{-7}, 1 \times 10^{-6}$, and $5 \times 10^{-6} \mu \mathrm{M}^{-2} \mathrm{~s}^{-1}$, corresponding to spontaneous, capping-protein-stimulated or formin-stimulated actin nucleation respectively [newly created filaments are assumed to contain three actin protomers (Sept and McCammon 2001)]. Rate constants for the simulation of the stimulated nucleation are pseudo parameters and calculated as a product of the actual rate constant (Carlsson et al. 2004; Pring et al. 2003; Sept and McCammon 2001) and the corresponding concentration of capping protein or formin $(\sim 0.1 \mu \mathrm{M})$. Results of the actin turnover simulations are shown in Fig. $1 \mathrm{~b}$ and represent the average filament length as a function of time. The average length increased during the first phase and reached steadystate levels of 4200, 1400, and 600 subunits in accordance with the parameters $k_{\text {nuc }}=1 \times 10^{-7}, 1 \times 10^{-6}$, and $5 \times 10^{-6} \mu \mathrm{M}^{-2} \mathrm{~s}^{-1}$. Decrease of the steady-state average length by increasing the nucleation rate is caused by the growing number of the nucleated filaments during earlier polymerisation phase. When the G-actin concentration is below $1.0 \mu \mathrm{M}$, the probability of actin nucleation becomes nearly negligible and turnover is due to the gain/loss of actin subunits at filament ends. In our simulations, the steady-state G-actin concentration was $0.86 \mu \mathrm{M}$ (the initial G-actin concentration for all simulations was $6.0 \mu \mathrm{M}$ ), which is invariant by the nucleation rates (Fig. $1 \mathrm{~b}$; inset). On the other hand, the value $0.86 \mu \mathrm{M}$ appeared to be equal to the calculated critical concentration using Eq. 12 and the parameters of association/dissociation reactions used for simulations: $k_{b}^{+}=10.0 \mu \mathrm{M}^{-1} \mathrm{~s}^{-1}, \quad k_{b}^{-}=1.0 \mathrm{~s}^{-1}$, $k_{p}^{+}=0.5 \mu \mathrm{M}^{-1} \mathrm{~s}^{-1}, k_{p}^{-}=8.0 \mathrm{~s}^{-1}$. The obtained steadystate data and corresponding parametric configurations were further used to simulate the corresponding FRAP curves.

The FRAP curves were modelled for three average lengths of filaments at steady state, corresponding to various nucleation rates. The corresponding fits by the kinetic model are shown in Fig. 2a. Good fits were obtained indicating that our developed kinetic model could reproduce the results of stochastic simulations within an acceptable statistical error range $(<5 \%)$. Being in excellent agreement as proved by the two modelling methods, our synthetic data showed an interesting and new effect of an actin turnover studied by fluorescence recovery: the FRAP curves were found to behave as a function of filament length, exhibiting faster recovery rates for systems with a short average filament length and slower recovery rates for systems with relatively long filaments. Importantly, we theoretically confirm experimental findings on accelerating the actin turnover in the presence of actin nucleating proteins (Lai et al. 2008). Additionally, the simulated fluorescence recovery shown in Fig. 2a exhibited a linear curve. Linearity is caused by the relatively high elongation rate $v=7.6 \mathrm{sub} \mathrm{s}^{-1}$ and the diffusivity coefficients 4.8 and $4.2 \mathrm{sub}^{2} \mathrm{~s}^{-1}$ for BE and PE respectively (see Eq. 13). The FRAP curve is nonlinear when the absolute value of the elongation rate $v<0.5 \mathrm{sub} \mathrm{s}^{-1}$ (e.g. Fig. 2c).

To examine the effect of actin association and/or dissociation at either filament end on the FRAP response, we computed fluorescence recovery using the developed kinetic model for a range of the elongation coefficient $v$, from 0.1 up to $10 \mathrm{sub} \mathrm{s}^{-1}$, keeping constant values for other systems parameters: $\quad D_{b}=30.0 \mathrm{sub}^{2} \mathrm{~s}^{-1}, \quad D_{p}=5.0 \mathrm{sub}^{2} \mathrm{~s}^{-1}$, $c_{m}=0.17 \mu \mathrm{M}, c_{f}=1.0 \mu \mathrm{M}, L=1000 \mathrm{sub}$. The results of the kinetic modelling are presented in Fig. $2 b$. The predicted FRAP curves differed noticeably, indicating strong dependence on the fitting parameter $v$. As noted above, values of the elongation rate $v$ below 0.5 sub s$~_{-1}$ led to the non-linear 

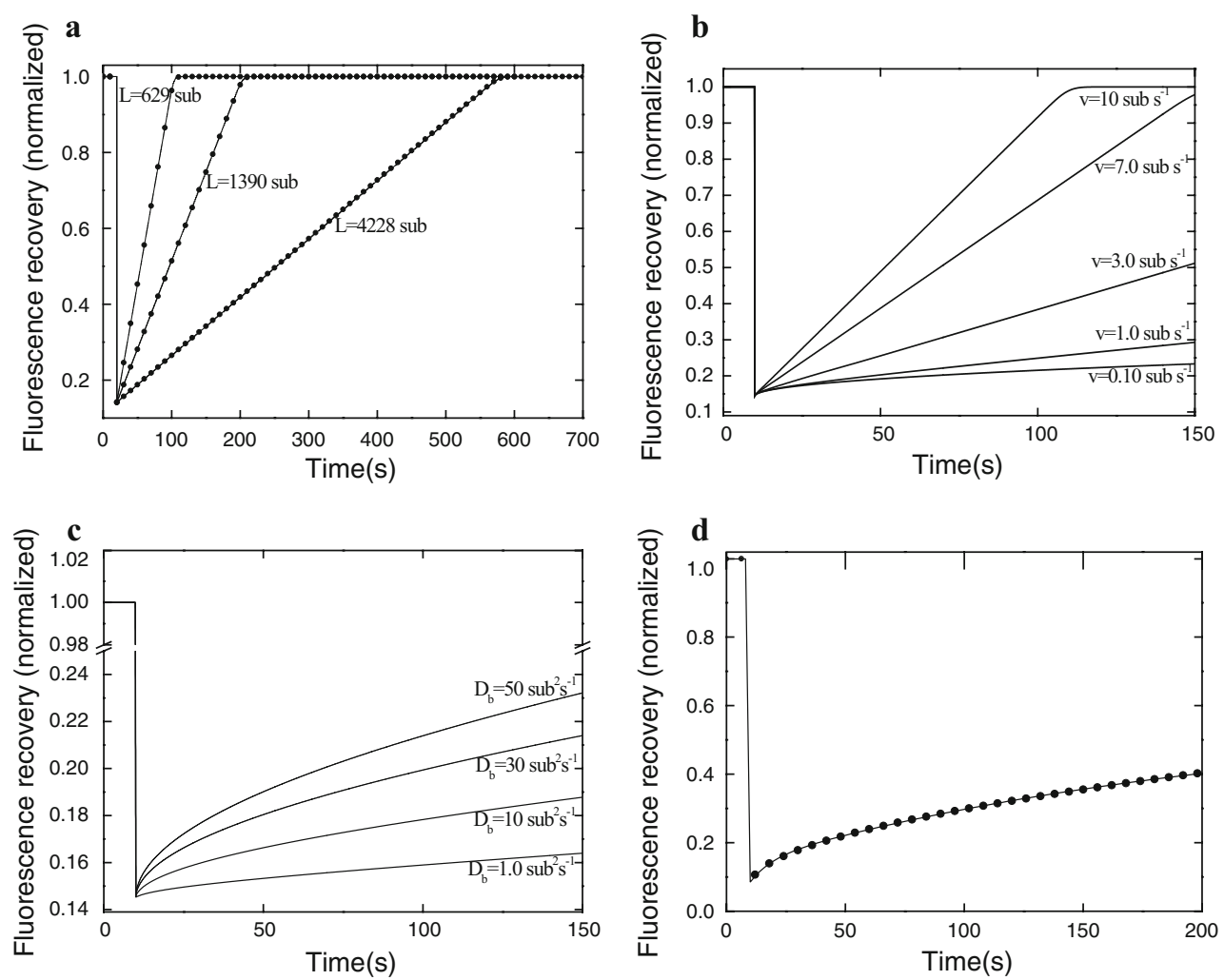

Fig. 2 Model calculations of the FRAP curves for actin filaments under steady-state conditions. a Predicted FRAP using analytic (line) and stochastic simulation (dot) methods for different values of the average filament length $\langle L\rangle$, corresponding to the steady-state mean lengths in Fig. $1 \mathrm{~b}$ (slower recovery at the smaller nucleation rate), with parameters $k_{b}^{+}=10.0 \mu \mathrm{M}^{-1} \mathrm{~s}^{-1}, k_{b}^{-}=1.0 \mathrm{~s}^{-1}, k_{p}^{+}=0.5 \mu \mathrm{M}^{-1} \mathrm{~s}^{-1}$, $k_{p}^{-}=8.0 \mathrm{~s}^{-1}, c_{f}=5.14 \mu \mathrm{M}$. Dimensionality of $\langle L\rangle$ is $1 \mathrm{sub}$ (subunit, $\sim 2.7 \mathrm{~nm}$ ). b Predicted FRAP for a range of elongation rates $v$. Other kinetic parameters are $D_{b}=30.0 \mathrm{sub}^{2} \mathrm{~s}^{-1}, D_{p}=5.0 \mathrm{sub}^{2} \mathrm{~s}^{-1}$,

curve and slow recovery. An increase in the parameter $v$, indicating an acceleration of treadmilling, resulted in the nearly linear increase of the FRAP intensity. Fluctuations of the filament length, controlled by the diffusivity coefficients $D_{b}$ and $D_{p}$ for low $v$ values, had a weaker effect on the FRAP curves, as shown in Fig. 2c, and yielded a non-linear recovery. We conclude that the FRAP curves may exhibit both a linear and non-linear behaviour, depending substantially on the elongation rate $v$.

Finally, we investigated crossover of the developed kinetic model and the existing FRAP fitting models. The only match resulting in acceptable fits (an error $<5 \%$ ) was obtained when compared with a two-binding state model interpreted as the outcome of the dynamically active PE and $\mathrm{BE}$ of filament and when our model was launched in the "non-linear" regime of the kinetic parameters $v, D_{b}$ and $D_{p}$ (Fig. 2d). As follows from Eq. 14 the sum of diffusivity coefficients $D_{b}$ and $D_{p}$ is numerically equal to the sum of dissociation rate constants $k_{b}^{-}$and $k_{p}^{-}$multiplied by the distance between neighbouring subunits in filament $\delta=1$ sub.

$c_{m}=0.17 \mu \mathrm{M}, c_{f}=1.0 \mu \mathrm{M}, L=1000$ sub. c Predicted FRAP for a range of diffusivity coefficients $D_{b}$. Other kinetic parameters are $D_{p}=0.1 \mathrm{sub}^{2} \mathrm{~s}^{-1}, v=0.1 \mathrm{sub} \mathrm{s}^{-1}, \quad c_{m}=0.17 \mu \mathrm{M}, \quad c_{f}=1.0 \mu \mathrm{M}$, $L=1000$ sub. d Predicted FRAP using the two-binding state (line) (Sprague et al. 2004) and the polymerisation (dot) models. Parameters of the polymerisation model are $D_{b}=5.0 \mathrm{sub}^{2} \mathrm{~s}^{-1}, D_{p}=5.0 \mathrm{sub}^{2} \mathrm{~s}^{-1}$, $c_{m}=0.1 \mu \mathrm{M}, \quad c_{f}=2.0 \mu \mathrm{M}, L=200 \mathrm{sub}, \quad v=0.1 \mathrm{sub} \mathrm{s}^{-1}$. Parameters of the two-binding model are $c_{1}=0.74 \mu \mathrm{M}, c_{2}=0.11 \mu \mathrm{M}$, $k_{\text {off } 1}=0.0049 \mathrm{~s}^{-1}, k_{\text {off } 2}=0.11 \mathrm{~s}^{-1}$

This fitting parameter characterises the total effective depolymerisation of filaments and is used herein as a measure for comparison of two models. As an example for the comparison test, we generated a synthetic dataset with parameters $\quad D_{b}=5.0 \mathrm{sub}^{2} \mathrm{~s}^{-1}, \quad D_{p}=5.0 \mathrm{sub}^{2} \mathrm{~s}^{-1}$, $c_{m}=0.1 \mu \mathrm{M}, c_{f}=2.0 \mu \mathrm{M}, L=200 \mathrm{sub}, v=0.1 \mathrm{sub} \mathrm{s}^{-1}$. Then we fitted the synthetic data by the two-binding state equation (Sprague et al. 2004). A sum of the unbinding rate constants obtained by fitting the two-binding state model is $0.12 \mathrm{~s}^{-1}$, which is inconsistent with the value set in simulations. Therefore, the unbinding coefficients obtained by the two-binding state model can not be accepted generally as accurate estimations of the dissociation rates of F-actin unless being used as a rough approximation in some comparative studies.

Our model perfectly agreed with stochastic simulations and resulted in more confident data fitting and accurate estimation of the kinetic parameters than those done by previously reported models (Sprague et al. 2004; Tardy et al. 1995). 
The presented model provides a relation between actin filament dynamics and the shape of FRAP curves. The application of our mathematical formalism for specific biological systems may require, however, an adaptation of the equations. For example, non-steady-state monomer concentration at the cell leading edge will cause various polymerisation rates at filament ends. In addition, special spatial ordering of filaments may require some modification of the function $\varphi\left(x_{0}\right)$, where higher values of $\varphi\left(x_{0}\right)$ should correspond to the parts of filaments situated inside the bleached spot. Our present results pave the way for future verifications of extensions of our approach by fitting experimental data and by modelling FRAP curves obtained in different areas in cells.

\section{Conclusion}

We have developed a mathematical model that will considerably improve analysis and interpretation of FRAP experiments carried out on actin-polymerisation assays. As proved by the Monte Carlo simulations, this model provides unbiased reaction rate constants at the pointed and barbed ends of filaments and the average length of filaments. Our findings demonstrate that: (1) FRAP is a function of the average filament length, (2) FRAP is faster at higher actin nucleation rates, (3) the transition from the nonlinear to linear FRAP curves is mainly caused by the increase of the elongation rate $v$, (4) in contrast to existing FRAP models, our fitting equation correctly estimates parameters of polymerisation processes in different regimes of actin polymerisation dynamics. In the future, the model will allow for studying parameters regulating actin polymerisation in vivo and help establish predictive or fitting models for actin polymerisation mechanisms in living cells.

Acknowledgments The work was supported by the Human Frontier Science Program Organization (RGP0058/2005). Aliaksandr Halavatyi was awarded an exchange grant of the European Science Foundation program "Frontiers of Functional Genomics" (Exchange grant 1,500$)$. P.V. Nazarov was awarded a mobility grant of the Fonds National de la Recherche (FNR), Luxembourg. A. Halavatyi and $\mathrm{Z}$. Al Tanoury are supported by a $\mathrm{PhD}$ grant of the MCESR and the FNR, Luxembourg.

\section{References}

Campbell JJ, Knight MM (2007) An improved confocal FRAP technique for the measurement of long-term actin dynamics in individual stress fibers. Microsc Res Tech 70:1034-1040

Carlsson AE, Wear MA, Cooper JA (2004) End versus side branching by Arp2/3 complex. Biophys J 86:1074-1081

Carrero G, McDonald D, Crawford E, de Vries G, Hendzel MJ (2003) Using FRAP and mathematical modeling to determine the in vivo kinetics of nuclear proteins. Methods 29:14-28
Carrero G, Crawford E, Hendzel MJ, de Vries G (2004) Characterizing fluorescence recovery curves for nuclear proteins undergoing binding events. Bull Math Biol 66:1515-1545

Dunn GA (2002) Fluorescence localization after photobleaching (FLAP): a new method for studying protein dynamics in living cells. J Microsc 205:109-112

Ellenberg J, Siggia ED, Moreira JE, Smith CL, Presley JF, Worman HJ, Lippincott-Schwartz J (1997) Nuclear membrane dynamics and reassembly in living cells: targeting of an inner nuclear membrane protein in interphase and mitosis. $\mathrm{J}$ Cell Biol 138:1193-1206

Fass J, Pak C, Bamburg J, Mogilner A (2008) Stochastic simulation of actin dynamics reveals the role of annealing and fragmentation. J Theor Biol 252:173-183

Fujiwara I, Takahashi S, Tadakuma H, Funatsu T, Ishiwata S (2002) Microscopic analysis of polymerization dynamics with individual actin filaments. Nat Cell Biol 4:666-673

Gadgil C, Lee CH, Othmer HG (2005) A stochastic analysis of firstorder reaction networks. Bull Math Biol 67:901-946

Gardiner CW (2004) Handbook of stochastic methods for physics, chemistry and natural sciences, 3rd edn. Springer, Heidelberg

Giganti A, Friederich E (2003) The actin cytoskeleton as a therapeutic target: state of the art and future directions. Prog Cell Cycle Res 5:511-525

Giganti A, Plastino J, Janji B, Van Troys M, Lentz D, Ampe C, Sykes C, Friederich E (2005) Actin-filament cross-linking protein Tplastin increases Arp2/3-mediated actin-based movement. J Cell Sci 118:1255-1265

Halavatyi AA, Nazarov PV, Medves S, van Troys M, Ampe C, Yatskou M, Friederich E (2009) An integrative simulation model linking major biochemical reactions of actin-polymerization to structural properties of actin filaments. Biophys Chem 140:24 34

Kuhn JR, Pollard TD (2005) Real-time measurements of actin filament polymerization by total internal reflection fluorescence microscopy. Biophys J 88:1387-1402

Lai FP, Szczodrak M, Block J, Faix J, Breitsprecher D, Mannherz HG, Stradal TE, Dunn GA, Small JV, Rottner K (2008) Arp2/3 complex interactions and actin network turnover in lamellipodia. EMBO J 27:982-992

Lan Y, Papoian GA (2008) The stochastic dynamics of filopodial growth. Biophys J 94:3839-3852

Matzavinos A, Othmer HG (2007) A stochastic analysis of actin polymerization in the presence of twinfilin and gelsolin. $\mathrm{J}$ Theor Biol 249:723-736

McDonald D, Carrero G, Andrin C, de Vries G, Hendzel MJ (2006) Nucleoplasmic $\beta$-actin exists in a dynamic equilibrium between low-mobility polymeric species and rapidly diffusing populations. J Cell Biol 172:541-552

McGrath JL, Tardy Y, Dewey CF Jr, Meister JJ, Hartwig JH (1998) Simultaneous measurements of actin filament turnover, filament fraction, and monomer diffusion in endothelial cells. Biophys $\mathbf{J}$ 75:2070-2078

Mogilner A (2009) Mathematics of cell motility: have we got its number? J Math Biol 58:105-134

Mueller F, Wach P, McNally JG (2008) Evidence for a common mode of transcription factor interaction with chromatin as revealed by improved quantitative fluorescence recovery after photobleaching. Biophys J 94:3323-3339

Phair RD, Misteli T (2000) High mobility of proteins in the mammalian cell nucleus. Nature 404:604-609

Pollard TD, Borisy GG (2003) Cellular motility driven by assembly and disassembly of actin filaments. Cell 112:453-465

Pring M, Evangelista M, Boone C, Yang C, Zigmond SH (2003) Mechanism of formin-induced nucleation of actin filaments. Biochemistry 42:486-496 
Roland J, Berro J, Michelot A, Blanchoin L, Martiel JL (2008) Stochastic severing of actin filaments by actin depolymerizing factor/cofilin controls the emergence of a steady dynamical regime. Biophys J 94:2082-2094

Sept D, McCammon JA (2001) Thermodynamics and kinetics of actin filament nucleation. Biophys J 81:667-674

Soumpasis DM (1983) Theoretical analysis of fluorescence photobleaching recovery experiments. Biophys J 41:95-97

Sprague BL, Pego RL, Stavreva DA, McNally JG (2004) Analysis of binding reactions by fluorescence recovery after photobleaching. Biophys J 86:3473-3495
Tardy Y, McGrath JL, Hartwig JH, Dewey CF (1995) Interpreting photoactivated fluorescence microscopy measurements of steady-state actin dynamics. Biophys J 69:1674-1682

van Kampen NG (2007) Stochastic processes in physics and chemistry, 3rd edn. Elsevier, Amsterdam

Van Troys M, Huyck L, Leyman S, Dhaese S, Vandekerkhove J, Ampe C (2008) Ins and outs of ADF/cofilin activity and regulation. Eur J Cell Biol 87:649-667 